\title{
MORITA CONTEXTS OF ENRICHED CATEGORIES
}

\section{J. FISHER-PALMQUIST AND P. H. PALMQUIST}

ABSTRACT. Categories enriched over a closed category $\mathbf{V}$ are considered. The theorems and proofs are nonadditive while specializing when $\mathrm{V}$ is the category of abelian groups to yield different interpretations and proofs of old results. V-adjoint equivalences of certain Vfunctor categories are shown to correspond to generalized Morita equivalences between small V-categories. Morita contexts are given a simple description as certain cospans and are shown to support a 2-dimensional structure.

For a bicomplete closed category V $[6$, p. 180] we show that our generalized $\mathbf{V}$-Morita equivalences between small $\mathbf{V}$-categories correspond to $\mathbf{V}$ adjoint equivalences between the corresponding $\mathbf{V}$-functor categories. $\mathbf{V}$ Morita equivalences are defined as Morita contexts invertible with respect to horizontal composition. V-Morita contexts are a special kind of diagram $\left(\mathrm{C}_{1} \rightarrow \mathrm{C} \leftarrow \mathrm{C}_{0}\right)$ in the category of small $\mathbf{V}$-categories with horizontal composition induced by pushouts. In the classical case when $\mathbf{V}$ is the category of abelian groups and $\mathbf{C}_{0}$ and $\mathbf{C}_{1}$ are each additive categories with one object, our definition of Morita context is equivalent to that of Bass [1], [2]. Our point of view is to consider Morita contexts as arrows in a bicategory (à la Bénabou [3, pp. 3-6]) and to apply a morphism which takes a Morita context into a left adjoint. We use right Kan extensions [4] to express our basic constructions.

We use two special $\mathbf{V}$-categories $\mathbf{G}$ and 2 , each of which has $\{0,1\}$ as its set of objects, such that $\mathbf{G}(i, j)=I$ the unit object of $\mathbf{V}$ and $2(i, j)$ is the terminal object of $\mathbf{V}$ for all $i, j$. (If $\mathbf{V}$ is cartesian closed, $\mathbf{G}=2$.) The $\mathrm{V}$-category $[I]$ is the one object category with hom object $I$.

A V-Morita context $M$ is defined to be a pair $(C, T: C \rightarrow 2)$ where $\mathbf{C}$

Presented to the Society, January 25, 1973 under the title Morita contexts and the Brauer group of a closed category; received by the editors July 20,1973 and, in revised form, February 26, 1974.

AMS (MOS) subject classifications (1970). Primary 18D20, 18D25; Secondary 18A25, 18D05.

Key words and phrases. Closed category, enriched category, functor category, adjoint equivalence, Kan extension, cospan, dense, bicategory, 2-dimensional category, Morita context, Morita equivalence, atom. 
is a small $\mathbf{V}$-category and $T$ is a $\mathbf{V}$-functor, and a map of Morita contexts $F:(\mathbf{C}, T) \rightarrow\left(\mathbf{C}^{\prime}, T^{\prime}\right)$ is a $\mathbf{V}$-functor $F: \mathbf{C} \rightarrow \mathbf{C}^{\prime}$ such that $T^{\prime} F=T$. In other words, a Morita context $M=(\mathrm{C}, T)$ is a cospan $\left(\mathrm{C}_{1} \stackrel{d_{1}}{\longrightarrow} \mathrm{C} \stackrel{d_{0}}{\left.\stackrel{\mathrm{C}_{0}}{\longrightarrow}\right)}\right.$ in the category of small $\mathbf{V}$-categories [2] in which $d_{0}$ and $d_{1}$ are the inclusions of the corresponding fibers of $T$ and the set of objects of $\mathbf{C}$ is the disjoint union of the sets of objects of $\mathbf{C}_{0}$ and $C_{1}$, and a map of Morita contexts is a map of cospans. Morita contexts and the ir maps form a category $\mathbf{M}$ equipped with four important endofunctors. For $M=(\mathbf{C}, T)$, define the transpose of $M$ by $M^{t}=(\mathrm{C}, \tau T)$ where $\tau: 2 \rightarrow 2$ is given by $\tau(i)=j, i \neq j$; define the opposite of $M$ by $M^{\circ}=\left(\mathbf{C}^{\circ}, T^{\circ}\right)$. For a Morita context $M=\left(\mathbf{C}_{1} \rightarrow \mathbf{C} \leftarrow \mathbf{C}_{0}\right)$ define the left identity of $M$ by $l(M)=\left(\mathbf{C}_{1} \otimes \mathbf{G}, L\right)$ where $L$ is induced by projection on $\mathbf{G}$; define the right identity of $M$ by $x(M)=l\left(M^{t}\right)$.

If $M=\left(\mathbf{C}_{1} \rightarrow \mathbf{C} \leftarrow \mathbf{C}_{0}\right)$ and $M^{\prime}=\left(\mathbf{C}_{1}^{\prime} \rightarrow \mathbf{C}^{\prime} \leftarrow \mathbf{C}_{0}^{\prime}\right)$ are Morita contexts with $r(M)=l\left(M^{\prime}\right)$, define the $*$-composite $M * M^{\prime}$ by first taking the composite of the cospans, i.e., let

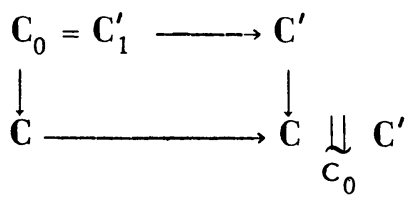

be a pushout in the category of small $\mathrm{V}$-categories and obtain the cospan $\left(\mathbf{C}_{1} \rightarrow \mathbf{C} \Perp_{C_{0}} \mathbf{C}^{\prime} \leftarrow \mathbf{C}_{0}^{\prime}\right)$, and then let the category of $M * M^{\prime}$ be the full subcategory of $\mathbf{C} \Perp_{C_{0}} C^{\prime}$ with objects the disjoint union of the objects of $C_{1}$ and $\mathbf{C}_{0}^{\prime}$. We note that for $X \in\left|\mathbf{C}_{0}^{\prime}\right|$ and $Z \in\left|\mathbf{C}_{1}\right|,\left(\mathbf{C} \|_{C_{0}} \mathbf{C}^{\prime}\right)(X, Z)$ is the coend [4] over all $Y \in\left|\mathbf{C}_{0}\right|$ of $\mathbf{C}(Y, Z) \otimes \mathbf{C}^{\prime}(X, Y)$. If $\phi: M \rightarrow N$ and $\phi^{\prime}$ : $M^{\prime} \rightarrow N^{\prime}$ are maps of Morita contexts such that $r(\phi)=l\left(\phi^{\prime}\right)$ then there is a map $\phi * \phi^{\prime}: M * M^{\prime} \rightarrow N * N^{\prime}$ by the universal property of pushouts. This $*$ composition is associative up to isomorphism since the composition of cospans is. There are left and right identity isomorphisms $l_{M}: l(M) * M \rightarrow M$ and $r_{M}$ :
$M * r(M) \rightarrow M$.

Theorem 1. If for small $\mathrm{V}$-categories $\mathrm{C}_{0}$ and $\mathrm{C}_{1}$ we define the category $\mathbf{B}\left(\mathbf{C}_{0}, \mathrm{C}_{1}\right)$ to have as objects Morita contexts $\left(\mathrm{C}_{1} \rightarrow \mathbf{C}-\mathrm{C}_{0}\right)$ and to have as maps only the maps of Morita contexts which are the identity on the fibers, then *-composition becomes a functor

$$
*: \mathrm{B}\left(\mathrm{C}_{1}, \mathrm{C}_{2}\right) \times \mathrm{B}\left(\mathrm{C}_{0}, \mathrm{C}_{1}\right) \rightarrow \mathrm{B}\left(\mathrm{C}_{0}, \mathrm{C}_{2}\right)
$$

and $\mathbf{B}$ is a bicategory in the sense of Bénabou [3, pp. 3-6].

Define maps $\lambda_{M}: M * M^{t} \rightarrow l(M)$ and $\rho_{M}: M^{t} * M \rightarrow \gamma(M)$ such that $\lambda_{M}$ 
is the identity on the fibers and $\lambda_{M}$ on the other hom objects is induced by the compositions

$$
\left\{\mathbf{C}(Y, Z) \otimes \mathbf{C}(X, Y) \rightarrow \mathrm{C}(X, Z) \mid Y \in \mathrm{C}_{0}\right\}
$$

and $\rho_{M}=\lambda_{M}$. Ignoring associativity and left and right identity isomorphisms we have equations:

$$
\begin{gathered}
\left(\lambda_{M} * M\right)=\left(M * \rho_{M}\right): M * M^{t} * M \rightarrow M ; \\
\lambda_{M * M^{\prime}}=\lambda_{M} \cdot\left(M * \lambda_{M^{\prime}} * M^{t}\right):\left(M * M^{\prime}\right) *\left(M * M^{\prime}\right)^{t} \rightarrow l(M) ; \\
\lambda_{N} \cdot\left(\phi * \phi^{t}\right)=l(\phi) \cdot \lambda_{M}: M * M^{t} \rightarrow l(N) ;
\end{gathered}
$$

for Morita contexts $M, M^{\prime}$, and $N$ with $r(M)=l\left(M^{\prime}\right)$ and $\phi: M \rightarrow N$ a map of Morita contexts.

If $\mathbf{V}$ is the category of abelian groups, the correspondence between our Morita contexts and those of Newell [7] which are 4-tuples $(U, V, \mu, \nu)$ is given as follows: $M=\left(\mathbf{C}_{1} \rightarrow \mathbf{C} \leftarrow \mathbf{C}_{0}\right)$ corresponds to the 4-tuple with $U$ (respectively, $V$ ) the restriction of the enriched hom of $\mathbf{C}$ to $\mathbf{C}_{0}^{0} \otimes \mathbf{C}_{1}$ (respectively, $\mathbf{C}_{1}^{0} \otimes \mathbf{C}_{0}$ ) and $\mu$ and $\nu$ the transformations induced by $\rho_{M}$ and $\lambda_{M}$, respectively.

Theorem 2. The following statements are equivalent for a Morita context M.

(a) There exists a Morita context $M^{\prime}$ such that $M * M^{\prime} \simeq l(M)$, i.e., $M$ has a fight *-inverse.

(b) $\lambda_{M}: M * M^{t} \rightarrow \not l(M)$ is a split epimorphism.

(c) $\lambda_{M}$ is an isomorphism.

(d) $M^{t}$ has a left $*$-inverse.

(e) $M^{\circ}$ has a right $*$-inverse.

Proof. The only hard part is (a) $\Rightarrow(b) \Rightarrow(c)$. To show (a) $\Rightarrow$ (b) we note that if $\phi: M * M^{\prime} \rightarrow l(M)$ is the isomorphism then equations (2) and (3) above give

$$
\lambda_{l(M)} \cdot\left(\phi * \phi^{t}\right)=l(\phi) \cdot \lambda_{M} \cdot\left(M * \lambda_{M^{\prime}} * M^{t}\right) .
$$

Since $\lambda_{l(M)}$ is an isomorphism and so are $l(\phi)$ and $\phi * \phi^{t}$, we have $\lambda_{M}$ is a split epimorphism.

If (b) holds then there is an $s: l(M) \rightarrow M * M^{t}$ such that $\lambda_{M} s=\mathrm{id}_{l(M)}$. Equation (1) applied to both $M$ and $M^{t}$ gives us that $s \lambda_{M}=\left(\lambda_{M} s\right) * M * M^{t}$ if we ignore all associativity and left and right identity isomorphisms. Hence $s \lambda_{M}=\mathrm{id}_{M * M t}$. 
A Morita context is said to be a Morita equivalence if it has both a left and a right *-inverse. Examples are $l(M)$ and $r(M)$.

There is another binary operation $\square$ on Morita contexts (which we might call vertical composition) which is always defined and is associative and commutative up to isomorphism. Namely, if $M=\left(\mathbf{C}_{1} \rightarrow \mathbf{C} \leftarrow \mathbf{C}_{0}\right)$ and $N=$ $\left(\mathbf{D}_{1} \rightarrow \mathbf{D} \leftarrow \mathbf{D}_{0}\right)$ are Morita contexts $M \square N$ has as its category the full subcategory of $\mathbf{C} \otimes \mathbf{D}$ with fibers $\mathbf{C}_{1} \otimes \mathbf{D}_{1}$ and $\mathbf{C}_{0} \otimes \mathbf{D}_{0}$. For Morita contexts $M, M^{\prime}, N$ and $N^{\prime}$ such that $r(M)=l\left(M^{\prime}\right)$ and $r(N)=l\left(N^{\prime}\right)$ we have equations:

$$
\begin{aligned}
& (M \square N) *\left(M^{\prime} \square N^{\prime}\right)=\left(M * M^{\prime}\right) \square\left(N * N^{\prime}\right) ; \\
& (M \square N)^{t}=M^{t} \square N^{t} \quad \text { and } \quad \rho_{M \square N}=\rho_{M} \square \rho_{N^{*}}
\end{aligned}
$$

Theorem 3. Let $M$ be a Morita context.

(i) If $M$ is a Morita equivalence so are $M^{t}$ and $M^{\circ}$.

(ii) If $M$ and $M^{\prime}$ are Morita equivalences with $r(M)=l\left(M^{\prime}\right)$, then $M * M^{\prime}$ is a Morita equivalence.

(iii) If $M$ and $N$ have left *-inverses so does $M \square N$.

(iv) If $r(M)=r(N)=([I] \rightarrow[I] \otimes \mathbf{G} \leftarrow[I])$ and $M \square N$ is a Morita equivalence, then $M$ and $N$ are Morita equivalences.

Proof. Statements (i), (ii) and (iii) follow from Theorem 2 and equations (2) and (5). If the hypotheses of (iv) hold then equation (4) and the equalities $M=M \square r(N)$ and $N=r(M) \square N$ yield the equations $M \square N=(l(M) \square N) * M$ and $M \square N=(M \square l(N)) * N$, from which the conclusions of (iv) follow.

Let $\mathbf{L a d}$ be the 2-dimen sional category with objects small $\mathbf{V}$-categories $\mathbf{C}$ and $\mathbf{L a d}\left(\mathbf{C}, \mathbf{C}^{\prime}\right)$ the category of $\mathbf{V}$-functors from $V^{C}$ to $\mathbf{V}^{\prime}$ which are $\mathbf{V}$ left adjoints, i.e., $\mathrm{V}$-cocontinuous, with maps $\mathrm{V}$-natural transformations. There is a strict homomorphism of bicategories $\Phi: \mathbf{B} \rightarrow$ Lad defined by

$$
\Phi(\mathrm{C})=\mathrm{C} \text { and } \Phi\left(\mathrm{C}_{1} \stackrel{d_{1}}{\longrightarrow} \mathrm{C} \stackrel{d_{0}}{\longleftarrow} \mathrm{C}_{0}\right)=\operatorname{Ran}_{\mathrm{v}^{d_{0}}} \mathrm{~V}^{d_{1}}=\mathrm{V}^{d_{1}} \cdot\left(\mathrm{V}^{d_{0}}\right)^{l},
$$

where we have computed the right Kan extension in terms of $\left(\mathbf{V}^{d} 0\right)^{l}$, the left adjoint of $\mathbf{V}^{d} 0$. Note that

$$
\Phi(M)\left(\mathrm{C}_{0}(X,-)\right)(Y)=\mathbf{C}(X, Y)
$$

for $X$ in $\mathbf{C}_{0}$ and $Y$ in $\mathbf{C}_{1}$. We then have natural transformations

and

$$
\Phi\left(\lambda_{M}\right): \Phi(M) \cdot \Phi\left(M^{t}\right) \rightarrow \Phi(l(M))=\mathrm{id}_{\mathrm{V}} \mathrm{c}_{1}
$$

$$
\Phi\left(\rho_{M}\right): \Phi\left(M^{t}\right) \cdot \Phi(M) \rightarrow \Phi(r(M))=\mathrm{id}_{\mathrm{V}} \mathrm{C}_{0}
$$


and for $X$ and $Z$ in $\mathrm{C}_{0}$

$$
\Phi\left(\rho_{M}\right)\left(\mathbf{C}_{0}(X,-)\right)(Z)=\rho_{M}(X, Z) .
$$

Theorem 4. If

$$
M=\left(\mathrm{C}_{1} \stackrel{d_{1}}{\longrightarrow} \mathrm{C} \stackrel{d_{0}}{\longleftarrow} \mathrm{C}_{0}\right)
$$

is a Morita context with a right *-inverse, then the following are true:

(i) $\Phi\left(M^{t}\right)$ is left adjoint to $\Phi(M)$ with counit $\Phi\left(\rho_{M}\right)$ and unit $\Phi\left(\lambda_{M}\right)^{-1}$ which is an isomorphism.

(ii) The functor $\Phi\left(M^{t}\right)$ maps V-atoms [5, (4.3)] into V-atoms and hence representables into $\mathbf{V}$-atoms.

(iii) The functor

$$
\mathrm{C}^{\mathrm{o}} \stackrel{R}{\rightarrow} \mathrm{V}^{\mathrm{C}} \stackrel{\mathrm{v}^{d}}{\longrightarrow} \mathrm{V}^{\mathrm{C}} \mathrm{C}_{\mathrm{O}}
$$

where $R$ is the Yoneda embedding, is V-full and faithful, i.e., $d_{0}: \mathbf{C}_{0} \rightarrow \mathbf{C}$ is $\mathbf{V}$-codense [4].

(iv) $M$ has a left *-inverse if and only if

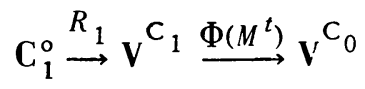

is $\mathbf{V}$-dense.

Proof. (i) is a consequence of applying $\Phi$ which is a strict map of bicategories to equation (1) for $M$ and $M^{t}$. (ii) follows from the fact that $\Phi(M)$ is the right adjoint of $\Phi\left(M^{t}\right)$ and is $\mathrm{V}$-cocontinuous. Thus for $G$ a $\mathbf{V}$-atom in $\mathbf{V}^{\mathrm{C}_{1}}$, we have

$$
\mathrm{V}^{\mathrm{C}_{0}}\left(\Phi\left(M^{t}\right) G,-\right) \simeq \mathrm{V}^{\mathrm{C}_{1}}(G,-) \cdot \Phi(M)
$$

which is $\mathbf{V}$-cocontinuous. Part (iii) is equivalent to stating both that

$$
\mathrm{C}_{1}^{\mathrm{o}} \stackrel{R}{\rightarrow} \mathrm{V}^{\mathrm{C}_{1}} \stackrel{\Phi\left(M^{t}\right)}{\longrightarrow} \mathrm{V} \mathrm{C}_{0}
$$

is V-full and faithful, which is true since $R_{1}$ and $\Phi\left(M^{t}\right)$ are, and that

$$
\Phi(M)\left(\mathrm{C}_{0}\left(C_{0},-\right)\right) C_{1}=\mathrm{V}^{\mathrm{C}_{0}}\left(\Phi\left(M^{t}\right) R_{1}\left(C_{1}\right), \mathrm{C}_{0}\left(C_{0},-\right)\right)
$$

for $C_{0}$ in $\mathbf{C}_{0}$ and $C_{1}$ in $\mathbf{C}_{1}$, which holds since $\Phi(M)$ is $\mathbf{V}$-right adjoint to $\Phi\left(M^{t}\right)$.

To show (iv) we note that since $\Phi\left(M^{t}\right)$ is V-cocontinuous, $\Phi\left(M^{t}\right) \cdot R_{1}$ is $\mathbf{V}$-dense if and only if $\Phi\left(M^{t}\right)$ is $\mathbf{V}$-dense. But $\Phi\left(M^{t}\right)$ is $\mathbf{V}$-dense if and 
only if its right adjoint is full and faithful, i.e., $\Phi\left(\rho_{M}\right)$ is an isomorphism. But by (6) $\Phi\left(\rho_{M}\right)$ is an isomorphism if and only if $\rho_{M}$ is one.

Corollary 5. The map of bicategories $\Phi: \mathbf{B} \rightarrow$ Lad induces an isomorphism of the Picard groupoids [3, p. 57$]$

$$
\hat{\Phi}: \text { Pic B } \rightarrow \text { Pic (Lad). }
$$

(Pic $B)\left(C_{0}, C_{1}\right)$ is the set of is omorphism classes of Morita equivalences, i.e., invertible arrows from $C_{0}$ to $C_{1}$.

\section{REFERENCES}

1. H. Bass, Algebraic K-theory, Benjamin, New York, 1968. MR 40 \#2736.

2. - The Morita theorems, University of Oregon (mimeographed notes).

3. J. Bénabou, Introduction to bicategories, Reports of the Midwest Category Seminar, Lecture Notes in Math., vol. 47, Springer-Verlag, Berlin and New York, 1967, pp. 1-77. MR $36 \# 3841$.

4. E. Dubuc, Kan extensions in enriched category theory, Lecture Notes in Math., vol. 145, Springer-Verlag, Berlin and New York, 1970. MR 43 \#6280.

5. J. Fisher-Palmquist and D. C. Newell, Triples on functor categories, J. Algebra 25 (1973), 226-258. MR 48 \#2218.

6. S. Mac L ane, Categories for the working mathematician, Springer-Verlag, New York, 1971.

7. D. C. Newell Morita theorems for functor categories, Trans. Amer. Math. Soc. 168 (1972), 423-433. MR $45 \# 3512$

DEPARTMEN T OF MATHEMATICS, UNIVERSITY OF CALIFORNIA, IRVINE, CALIFORNIA 92664 